\title{
Clinical application of liquid biopsies to detect somatic BRCA1/2 mutations and guide potential therapeutic intervention for patients with metastatic breast cancer
}

\author{
Neelima Vidula ${ }^{1}$, Leif W. Ellisen ${ }^{1}$ and Aditya Bardia ${ }^{1}$ \\ ${ }^{1}$ Massachusetts General Hospital, Harvard Medical School, Boston, MA, USA \\ Correspondence to: Neelima Vidula, email: nvidula@mgh.harvard.edu \\ Keywords: cell-free DNA; BRCA 1/2; PARP inhibitor; metastatic breast cancer; plasma based genotyping \\ Received: November 19, $2020 \quad$ Accepted: December 22, $2020 \quad$ Published: January 19, 2021 \\ Copyright: @ 2021 Vidula et al. This is an open access article distributed under the terms of the Creative Commons Attribution License (CC \\ BY 3.0), which permits unrestricted use, distribution, and reproduction in any medium, provided the original author and source are credited.
}

\begin{abstract}
Plasma based genotyping via cell-free DNA may identify actionable mutations for potential therapeutic intervention in patients with advanced malignancies including breast cancer. In this article, we discuss recent studies using cell-free DNA testing to identify and classify somatic BRCA1/2 mutations in metastatic breast cancer, and potential future applications for the treatment of metastatic breast cancer.
\end{abstract}

\section{INTRODUCTION}

In recent years, plasma based genotyping via cellfree DNA (cfDNA) or "liquid biopsy" has emerged as a robust means to detect actionable mutations and guide genotype-directed therapies for patients with advanced malignancies [1]. For metastatic breast cancer, the utility of cfDNA in identifying actionable mutations has been demonstrated [2], and recent studies have validated the feasibility of tumor genotyping for targeted treatment matching [3]. Notably, a PI3K inhibitor has now been approved for PI3KCA mutant hormone receptor positive $(\mathrm{HR}+) /$ HER2- metastatic breast cancer using diagnostic cfDNA testing to identify PI3KCA mutations [4]. CfDNA offers the advantage of being less invasive and possibly more sensitive than tumor tissue genotyping assays $[5,6]$.

PARP inhibitors have recently been approved as a targeted therapy for the treatment of germline BRCA1/2 mutant metastatic breast cancer based on results from two pivotal phase III clinical trials. The phase III OlympiAD [7] and EMBRACA [8] studies demonstrated significant improvement in progression-free survival with olaparib and talazoparib, respectively, compared to chemotherapy, for metastatic breast cancer. PARP inhibitors may also improve patient reported outcomes and quality of life. However, germline $B R C A 1 / 2$ mutations only account for about $5-10 \%$ of breast cancer [9], limiting their broad applicability.

A question that arises is whether PARP inhibitors may also be beneficial in metastatic breast cancer with somatic BRCA1/2 mutations. In a recent study led by our team [10], we demonstrated the emergence of somatic $B R C A 1 / 2$ mutations detectable by cfDNA, largely in the absence of germline $B R C A 1 / 2$ mutations. In our analysis of 215 patients undergoing cfDNA testing as part of care for metastatic breast cancer, we observed that 29 (13.5\%) had somatic BRCA1/2 mutations detectable in cfDNA, which were seen in various subtypes, and often clonal in nature. Four percent had somatic $B R C A 1 / 2$ mutations that were known germline-pathogenic, and the rest were novel variants, based on classification using extrapolation from reputable genomic databases. In addition, we demonstrated increased sensitivity to a PARP inhibitor in a CTC culture derived from a patient with a pathogenic somatic BRCA1 mutation, highlighting the unique role of PARP inhibition for patients with somatic $B R C A 1 / 2$ mutant breast cancer. However, not all somatic $B R C A 1 / 2$ mutations are functionally significant, i.e., pathogenic. For example, in a CTC culture derived from a patient with a novel variant $B R C A 2$ mutation, we did not observe any impact of a PARP inhibitor. Interestingly, this patient also had widespread expression of the APOBEC mutation signature that encompassed the $B R C A 2$ mutation itself, suggesting the novel $B R C A 2$ mutation was likely a passenger mutation rather than a driver mutation. Based on these findings, we developed an algorithm to guide clinical assessment of somatic BRCA1/2 mutations, as well as designed a genotype-directed clinical trial for patients with metastatic breast cancer [11].

A genotype-directed clinical trial is currently ongoing at our institution and other academic centers to determine the efficacy of PARP inhibition in somatic 
cfDNA BRCA1/2-mutant metastatic breast cancer [11], and the results of this trial may help expand the population of patients who benefit from PARP inhibitors, similar to what has been observed in ovarian cancer. A combined analysis of two studies evaluating a PARP inhibitor in ovarian cancer demonstrated similar efficacy in germline $B R C A 1 / 2$ mutant patients and somatic BRCA1/2 mutant patients [12].

Besides detection of germline and somatic $B R C A 1 / 2$ mutations, cfDNA analysis also allows for detection of reversion $B R C A 1 / 2$ mutations [13]. The acquisition of $B R C A 1 / 2$ reversion mutations is a well described phenomenon [14], which restores the open reading frame (and function) of the BRCA1/2 gene, thus rendering a PARP inhibitor ineffective. In a second multicenter analysis [13], we demonstrated that routine plasma-based genotyping can be utilized to classify $B R C A 1 / 2$ cfDNA mutations as germline, somatic or reversion mutations, based on specific loci and the mutant allele fraction of the $B R C A 1 / 2$ mutation. Of 828 patients with advanced malignancies including breast cancer undergoing testing with a 73 gene cfDNA assay, one or more pathogenic $B R C A 1 / 2$ mutation was identified in $7.2 \%$ of patients, and both somatic and germline variants were detected. Polyclonal reversion mutations were found in $21.4 \%$ of patients with germline $B R C A 1 / 2$ mutations, most often in association with receipt of a prior PARP inhibitor.

Another study found that the absence of pre-existing cfDNA $B R C A 1 / 2$ reversion mutations in patients with ovarian cancer who had somatic or germline $B R C A 1 / 2$ mutations and were treated with rucaparib was associated with improved progression-free survival [15]. Therefore, the identification of cfDNA BRCA1/2 reversion mutations may have important implications for therapeutic response to PARP inhibitors, and will be studied in our ongoing clinical trial of a PARP inhibitor for somatic BRCA1/2 mutant metastatic breast cancer [11]. A complementary ongoing trial is also evaluating the efficacy of the PARP inhibitor, olaparib, in somatic BRCA1/2 mutant metastatic breast cancer, with initial results suggesting potential efficacy [16].

In summary, plasma-based genotyping is a promising strategy for the detection of $B R C A 1 / 2$ mutations and could potentially guide triage to genotype-directed matched therapy with a PARP inhibitor. Ongoing studies will help determine the therapeutic utility of this approach and impact on long term clinical outcomes for patients with metastatic breast cancer.

\section{Author contributions}

All authors contributed to writing this article.

\section{CONFLICTS OF INTEREST}

Neelima Vidula: Research grant funding (to the institution): Pfizer, Daehwa, Radius, Merck, Novartis;
Travel: Pfizer; Advisory Board: AbbVie. Aditya Bardia: Consulting/advisory board: Pfizer, Novartis, Genentech, Merck, Radius Health, Immunomedics, Taiho, Sanofi, Diiachi Pharma/Astra Zeneca, Puma, Biothernostics Inc., Phillips, Eli Lilly, Foundation Medicine; Contracted Research/Grant (to institution): Genentech, Novartis, Pfizer, Merck, Sanofi, Radius Health, Immunomedics, Diiachi Pharma/Astra Zeneca.

\section{REFERENCES}

1. Diaz LA Jr, Bardelli A. Liquid biopsies: genotyping circulating tumor DNA. J Clin Oncol. 2014; 32:579-86. https://doi.org/10.1200/jco.2012.45.2011. [PubMed]

2. Vidula N, Juric D, Niemierko A, Spring L, Moy B, Malvarosa G, Yuen M, Habin K, Shin JA, Peppercorn JM, Isakoff SJ, Ellisen LW, Iafrate AJ, et al. Comparison of tissue genotyping (TG) vs circulating tumor DNA (ctDNA) for selection of matched therapy and impact on clinical outcomes among patients with metastatic breast cancer (MBC). J Clin Oncol. 2018; 36:1020. https://doi. org/10.1200/JCO.2018.36.15 suppl.1020.

3. Turner N, Kingston B, Kilburn L, Kernaghan S, Wardley A, Macpherson I, Baird RD, Roylance R, Stephens P, Oikonomidou O, Braybrooke JP, Tuthill M, Abraham J, et al. Abstract GS3-06: Results from the plasmaMATCH trial: A multiple parallel cohort, multi-centre clinical trial of circulating tumour DNA testing to direct targeted therapies in patients with advanced breast cancer (CRUK/15/010). Cancer Res. 2020; 80:Abstract GS3-06. https://doi. org/10.1158/1538-7445.SABCS19-GS3-06.

4. Andre F, Ciruelos E, Rubovszky G, Campone M, Loibl S, Rugo H, Iwata H, Conte P, Mayer IA, Kaufman B, Yamashita T, Lu YS, Inoue K, et al. Alpelisib for PIK3CAMutated, Hormone Receptor-Positive Advanced Breast Cancer. N Engl J Med. 2019; 380:1929-40. https://doi. org/10.1056/NEJMoa1813904. [PubMed]

5. Haber DA, Velculescu VE. Blood-based analyses of cancer: circulating tumor cells and circulating tumor DNA. Cancer Discov. 2014; 4:650-61. https://doi.org/10.1158/2159-8290. cd-13-1014. [PubMed]

6. Somlo G, Lau SK, Frankel P, Hsieh HB, Liu X, Yang L, Krivacic R, Bruce RH. Multiple biomarker expression on circulating tumor cells in comparison to tumor tissues from primary and metastatic sites in patients with locally advanced/inflammatory, and stage IV breast cancer, using a novel detection technology. Breast Cancer Res Treat. 2011; 128:155-63. https://doi.org/10.1007/s10549-011-1508-0. [PubMed]

7. Robson M, Im SA, Senkus E, Xu B, Domchek SM, Masuda N, Delaloge S, Li W, Tung N, Armstrong A, Wu W, Goessl C, Runswick S, Conte P. Olaparib for Metastatic Breast Cancer in Patients with a Germline BRCA Mutation. N Engl J Med. 2017; 377:523-33. https://doi.org/10.1056/ NEJMoa1706450. [PubMed] 
8. Litton JK, Rugo HS, Ettl J, Hurvitz SA, Gonçalves A, Lee KH, Fehrenbacher L, Yerushalmi R, Mina LA, Martin M, Roché H, Im YH, Quek RG, et al. Talazoparib in Patients with Advanced Breast Cancer and a Germline BRCA Mutation. N Engl J Med. 2018; 379:753-63. https://doi. org/10.1056/nejmoa1802905. [PubMed]

9. Campeau PM, Foulkes WD, Tischkowitz MD. Hereditary breast cancer: new genetic developments, new therapeutic avenues. Hum Genet. 2008; 124:31-42. https://doi. org/10.1007/s00439-008-0529-1. [PubMed]

10. Vidula N, Dubash T, Lawrence M, Simoneau A, Niemierko A, Blouch E, Nagy B, Roh W, Chirn B, Reeves B, Malvarosa $\mathrm{G}$, Lennerz J, Isakoff $\mathrm{S}$, et al. Identification of Somatically Acquired BRCA1/2 Mutations by cfDNA Analysis in Patients with Metastatic Breast Cancer. Clin Cancer Res. 2020; 26:4852-62. https://doi.org/10.1158/1078-0432.ccr20-0638. [PubMed]

11. Evaluation of talazoparib, a PARP inhibitor, in patients with somatic BRCA mutant metastatic breast cancer: genotyping based clinical trial. https:/www.clinicaltrials.gov/ct2/show/ NCT03990896.

12. Oza AM, Tinker A, Oaknin A, Shapira-Frommer R, McNeish IA, Swisher EM, Ray-Coquard I, Bell-McGuinn K, Coleman R, O'Malley D, Leary A, Chen LM, Provencher $\mathrm{D}$, et al. Antitumor activity and safety of the PARP inhibitor rucaparib in patients with high-grade ovarian carcinoma and a germline or somatic BRCA1 or BRCA2 mutation: Integrated analysis of data from Study 10 and ARIEL2. Gynecol Oncol. 2017; 147:267-275. https://doi. org/10.1016/i.ygyno.2017.08.022. [PubMed]
13. Vidula N, Rich TA, Sartor O, Yen J, Hardin A, Nance T, Lilly M, Nezami M, Patel S, Carneiro B, Fan A, Brufsky A, Parker B, et al. Routine Plasma-Based Genotyping to Comprehensively Detect Germline, Somatic, and Reversion BRCA Mutations among Patients with Advanced Solid Tumors. Clin Cancer Res. 2020; 26:2546-55. https://doi. org/10.1158/1078-0432.ccr-19-2933. [PubMed]

14. Weigelt B, Comino-Mendez I, de Brujin I, Tian L, Meisel J, Garcia-Murillas I, Fribbens C, Cutts R, Martelotto L, Ng C, Lim R, Selenica P, Piscuoglio S, et al. Diverse BRCA1 and $B R C A 2$ Reversion Mutations in Circulating Cell-Free DNA of Therapy-Resistant Breast or Ovarian Cancer. Clin Cancer Res. 2017; 23:6708-20. https://doi.org/10.1158/1078-0432. ccr-17-0544. [PubMed]

15. Lin K, Harrell M, Oza A, Oaknin A, Ray-Coquard I, Tinker A, Helman E, Radke M, Say C, Vo LT, Mann E, Isaacson J, Maloney L, et al. BRCA Reversion Mutations in Circulating Tumor DNA Predict Primary and Acquired Resistance to the PARP Inhibitor Rucaparib in High-Grade Ovarian Carcinoma. Cancer Discov. 2019; 9:210-219. https://doi. org/10.1158/2159-8290.cd-18-0715. [PubMed]

16. Tung NM, Robson M, Ventz S, Santa-Maria C, Nanda R, Marcom P, Shah P, Ballinger T, Yang E, Vinayak S, Melisko M, Brufsky A, DeMeo M, et al. TBCRC 048: Phase II Study of Olaparib for Metastatic Breast Cancer and Mutations in Homologous Recombination-Related Genes. J Clin Oncol. 2020; 38:4274-4282. https://doi.org/10.1200/jco.20.02151. [PubMed] 